\title{
Pi64, Encoding a Novel CC-NBS-LRR Protein, Confers Resistance to Leaf and Neck Blast in Rice
}

\author{
Jian Ma, ${ }^{1}$ Cailin Lei, ${ }^{1}$ Xingtao Xu, ${ }^{1}$ Kun Hao, ${ }^{1}$ Jiulin Wang, ${ }^{1}$ Zhijun Cheng, ${ }^{1}$ Xiaoding Ma, ${ }^{1}$ Jin Ma, ${ }^{1}$ \\ Kunneng Zhou, ${ }^{2}$ Xin Zhang, ${ }^{1}$ Xiuping Guo, ${ }^{1}$ Fuqing Wu, ${ }^{1}$ Qibing Lin, ${ }^{1}$ Chunming Wang, ${ }^{2}$ Huqu Zhai, ${ }^{1}$ \\ Haiyang Wang, ${ }^{1}$ and Jianmin Wan ${ }^{1,2}$ \\ ${ }^{1}$ Institute of Crop Science, Chinese Academy of Agricultural Sciences/National Key Facility for Crop Gene Resources and \\ Genetic Improvement, Beijing 100081, China; ${ }^{2}$ National Key Laboratory of Crop Genetics and Germplasm Enhancement / \\ Jiangsu Provincial Center of Plant Gene Engineering, Nanjing Agricultural University, Nanjing 210095, China
}

Submitted 14 November 2014. Accepted 10 December 2014.

Rice blast caused by Magnaporthe oryzae poses a major threat to rice production worldwide. The utilization of host resistance $(R)$ genes is considered to be the most effective and economic means to control rice blast. Here, we show that the japonica landrace Yangmaogu (YMG) displays a broader spectrum of resistance to blast isolates than other previously reported broad-spectrum resistant (BSR) cultivars. Genetic analysis suggested that YMG contains at least three major $R$ genes. One gene, Pi64, which exhibits resistance to indica-sourced isolate CH43 and several other isolates, was mapped to a 43-kb interval on chromosome 1 of YMG. Two open reading frames (NBS-1 and NBS-2) encoding nucleotide-binding site and leucine-rich repeat proteins were short-listed as candidate genes for Pi64. Constructs containing each candidate gene were transformed into three susceptible japonica cultivars. Only transformants with $N B S-2$ conferred resistance to leaf and neck blast, validating the idea that $N B S-2$ represents the functional Pi64 gene. Pi64 is constitutively expressed at all development stages and in all tissues examined. Pi64 protein is localized in both the cytoplasm and nucleus. Furthermore, introgression of Pi64 into susceptible cultivars via gene transformation and markerassisted selection conferred high-level and broad-spectrum leaf and neck blast resistance to indica-sourced isolates, demonstrating its potential utility in breeding BSR rice cultivars.

Plants have evolved elaborate defense systems to protect themselves against a wide range of pathogens, such as viruses, bacteria, fungi, nematodes, and insects. The defense responses mediated by disease resistance $(R)$ genes are triggered in plants when the product of a plant $R$ gene directly or indirectly recognizes the product of a specific pathogen avirulence (Avr) gene, and accordingly, the evolution of novel $R$ genes in plants serves to counteract the evolving Avr factors (Jones and Dangl 2006). These defense reactions are often accompanied by hypersensitive

J. Ma and C. Lei contributed to the work equally.

Corresponding authors: C. Lei; Telephone: +86-10-82105837;

E-mail: leicailin@caas.cn; and J. Wan; Telephone: +86-10-82108563; E-mail:wanjianmin@caas.cn

*The $\boldsymbol{e}$-Xtra logo stands for "electronic extra" and indicates that six supplementary figures, ten supplementary tables, and three supplementary data files are published online.

(C) 2015 The American Phytopathological Society responses (HR) (Zipfel 2008). To date, more than $70 R$ genes from different plant species have been cloned and characterized, and most of them encode NBS-LRR (nucleotide-binding site and leucine-rich repeats) proteins (Liu et al. 2007). Plant NBS-LRR proteins are divided into two classes based on their N-terminal domains. The toll and mammalian interleukine I receptor (TIR)NBS-LRR class contains the Drosophila TIR domain, whereas the non-TIR class most often contains a predicted coiled-coil (CC) domain at the N-terminus (Qi et al. 2012). The NBS domain is the most conserved region in NBS-LRR proteins and is probably involved in nucleotide binding, hydrolyzation, and control of cell death, whereas the LRR domain is generally required for specific recognition of pathogen effectors (Bryan et al. 2000; Martin et al. 2003; Zhou et al. 2006; Chen et al. 2011).

Magnaporthe oryzae infects rice plants at different growth stages, causing blast lesions on leaves, nodes, different parts of panicles, and grains. The disease caused by $M$. oryzae may appear in two main forms, leaf blast and neck (panicle) blast. Leaf blast can cause damage to the rice crop by reducing the green leaf area and, in severe cases, an entire planting can be destroyed. However, neck blast (known as panicle blast, a near synonym of panicle blast defined by the International Rice Research Institute), which infects around the panicle base or the upper-most internode or the lower part of the panicle axis (Chai and Jin 1995; Hao et al. 2009; International Rice Research Institute 2002; Li et al. 2010; Zhuang et al. 2002), usually is economically more important because a single infection near the panicle base can result in the complete loss of the panicle (Roumen 1992), and losses of up to $70 \%$ have been recorded in fields affected by neck blast (Puri et al. 2009). Some leaf blast-resistant cultivars are susceptible to neck blast and vice versa (Ishihara, et al. 2014; Puri et al. 2009; Sirithunya et al. 2002; Zhuang et al. 2002). This implies that the genetic mechanisms of host resistance might vary across growth stages (Zhuang et al. 2002). Tremendous efforts have been devoted to the identification and characterization of blast resistant rice cultivars over the past few decades, and more than 70 blast $R$ genes have been identified and mapped in rice (Liu et al. 2010; Zhu et al. 2012). Among them, 21 have been cloned and characterized, i.e., Pib, Pita, Pi9, Pi2/Piz-5, Piz-t, Pid2, Pi36, Pi37, Pik-m, Pit, Pi5, Pid3, Pi25, Pish, Pik, pi21, Pbl, Pia, Pik-h/Pi54, Pik-p, and Pil (www. ricedata.cn/gene/gene_pi.htm) (RoyChowdhury et al. 2012). Among the approximately 70 mapped blast $R$ genes, only Pi25(t) and $P b 1$, both encoding CC-NBS-LRR proteins, display resistance to neck and panicle blast, respectively (Hayashi et al. 2010; Wu et al. 2005; Zhuang et al. 2002). 
It is well-known that high pathogenic variability in $M$. oryzae often leads to rapid breakdown of resistant cultivars (Jia 2003; Zeigler et al. 1994). Thus, one strategy to develop broad-spectrum resistance is to pyramid multiple $R$ genes that, together, confer resistance to a wide array of $M$. oryzae isolates or races (Hittalmani et al. 2000). In this study, we report the cloning and characterization of a new blast-resistant gene, Pi64, from Yangmaogu (YMG), a japonica landrace from Yunnan province, China. We show that YMG has a broad blast-resistance spectrum against multiple Chinese $M$. oryzae isolates and contains at least three major $R$ genes. Map-based cloning revealed that Pi64 encodes a novel CC-NBS-LRR protein that is localized in both the cytoplasm and nucleus. Introduction of Pi64 into blast-susceptible rice cultivars conferred a high level of leaf and neck blast resistance to the indica-sourced $M$. oryzae isolate $\mathrm{CH} 43$, demonstrating its potential utility in breeding broadspectrum blast-resistant rice cultivars via either transgenic or molecular marker based selection and gene pyramiding.

\section{RESULTS}

\section{Responses of YMG to M. oryzae isolates.}

Cultivar YMG has been grown for several decades in China for its durable and high levels of resistance to blast throughout all growth stages (Collaborative Group for Identification of Yunnan Rice Genetic Resources for Resistance to Diseases and Pests 1992). To evaluate its blast response spectrum in more detail, cv. YMG and susceptible cv. Lijiangxintuanheigu (LTH, as a control), together with seven monogenic lines (ML), IRBL9-W， IRBLz5-CA，IRBLz-Fu，IRBL1-CL，IRBLb-B, IRBLzt-T and IRBLta-K1, were infected with a total of 557 $M$. oryzae isolatesfrom northeastern, northern, central, northwestern, southern, and southwestern China (Supplementary Table S1). Strikingly, YMG exhibited high-level resistance to all isolates and, thus, had a broader blast-resistance spectrum than several previously reported broad-spectrum resistant (BSR) cultivars, IRBL9-W, IRBLz5-CA, and IRBL1-CL, which harbor the well-known BSR genes Pi9, Pi2/Piz-5, and Pil, respectively (Liu et al. 2010; Wu et al. 2007; Zhu et al. 2012). This result suggested that YMG may contain additional blast $B S R$ genes.

\section{Genetic analysis of blast resistance in YMG.}

To understand the $R$ gene constitution of $\mathrm{YMG}, \mathrm{F}_{2}$, and $\mathrm{BC}_{1} \mathrm{~F}_{1}$ populations derived from the cross YMG $\times$ LTH were challenged with four different blast isolates, $\mathrm{CH} 43,1813-2,99$ 26-2, and HLJ07-1-1. With isolate $\mathrm{CH} 43$, the $\mathrm{F}_{2}$ population segregated in a $3 \mathrm{R}: 1 \mathrm{~S}$ ratio and the $\mathrm{BC}_{1} \mathrm{~F}_{1}$ population segregated in a 1R:1S ratio; with isolates 1813-2, 99-26-2, and HLJ07-1-1, the $F_{2}$ population segregated in a 15R:1S, 63R:1S, and 63R:1S ratios, respectively (Supplementary Table S2). These results indicated that the resistance to isolate $\mathrm{CH} 43$ in YMG is likely conferred by a single dominant gene, whereas the resistance to the other three isolates is probably controlled by either two or three dominant genes. Thus, YMG contains at least three $R$ genes, depending upon the particular $M$. oryzae isolate. The resistance gene to isolate $\mathrm{CH} 43$ was designated Pi64, following the recommended gene nomenclature system for rice (McCouch 2008).

\section{Mapping of Pi64.}

To determine the chromosome location of Pi64, 20 highly susceptible and 15 highly resistant $F_{2}$ individuals, derived from the cross $\mathrm{YMG} \times \mathrm{LTH}$, were genotyped using polymorphic markers selected from a total of 290 SSR (simple sequence repeat) and 212 insertion and deletion (InDel) markers (Lei et al. 2013; McCouch et al. 2002). We found that two SSR markers located on the long arm of chromosome 1, RM11715 and RM11787, were linked to the $R$ gene (Fig. 1A). Subsequently, 384 highly susceptible $F_{2}$ individuals derived from the same cross were genotyped, using five additional polymorphic markers (Fig. 1B; Supplementary Table S3). As a result, Pi64 was delimited to the Ty13-RM11747 interval on chromosome 1L (Fig. 1B). When an additional 1,900 susceptible $\mathrm{F}_{2}$ individuals were subjected to genotyping with 10 newly developed polymorphic markers (Fig. 1C), the location of Pi64 was narrowed to a 78.8-kb region on the BAC clone B1100D10 based on the Nipponbare sequence (Fig. 1D). This region contains 10 predicted open reading frames (ORF) based on the Nipponbare sequence and RiceGAAS program (International Rice Genome Sequencing Project 2005; Rice Genome Annotation Project database), including four tandemly repeated putative NBS-LRR-type genes, i.e., LOC_Os01g57270, LOC_Os01g57280, LOC_Os01g57310, and LOC_Os01g57340, within a 55-kb genomic interval called the Pish cluster (Takahashi et al. 2010) (Fig. 1D; Supplementary Table S4). Among the four NBS-LRR genes, LOC_Os01g57340 encodes the R protein Pish (Takahashi et al. 2010 ) and the allele of LOC_Os01g57310 in the rice cv. St. No.1 encodes the R protein Pi37 (Lin et al. 2007).

\section{Genomic sequencing of the Pi64 locus in YMG.}

To identify the candidate gene for Pi64 in YMG, the cleaved amplified polymorphic sequence 2 (CAPS2) to derived cleaved amplified polymorphic sequence 5 (dCAPS5) region of YMG was sequenced, based on the reference genome sequences of Nipponbare and 93-11 (Gramene database). The genome sequence assembly enabled the target CAPS2 to dCAPS5 region in YMG to be narrowed down to about $43 \mathrm{~kb}$ (Supplementary Data File S1). The 43-kb region of YMG contains seven ORF predicted by the online program FGENESH (Supplementary Table S5). Among them, only two NBS-LRR-type genes, ORF 13 and $O R F 16$, were identified, and they share highest nucleotide similarities to Nipponbare LOC_OsOlg57270 (59.1\%) and LOC_OsO1g57280 (93.20\%), respectively (Supplementary Table S6). This result indicated that $O R F 13$ and $O R F 16$ could be allelic to LOC_OsO1g57270 and LOC_OsO1g57280, respectively, and we tentatively named them NBS-1 (ORF13) and NBS-2 (ORF16) for convenience in later use (Fig. 1E).

\section{Complementation analysis of the Pi64 gene.}

To determine whether either NBS-1 or NBS-2, or both together, function as Pi64, we transformed separate genomic fragments containing the entire $N B S-1$ or entire $N B S-2$ under control of their native promoters into the susceptible japonica cultivars Fujisaka 5 and Tsuyuake by Agrobacterium tumefaciensmediated transformation. We found that all independent transgenic $\mathrm{T}_{0}$ lines carrying $N B S-1$ were still highly susceptible to isolate $\mathrm{CH} 43$ after injection-inoculation at the tillering stage (Supplementary Table S7). This indicated that NBS-1 was not responsible or not sufficient for Pi64-mediated resistance. On the contrary, all independent transgenic $\mathrm{T}_{0}$ lines carrying NBS-2 showed the same level of high resistance (disease score 0 ) as YMG when challenged with isolate $\mathrm{CH} 43$. We obtained 29 and 19 independent $N B S-2 \mathrm{~T}_{1}$ lines in Fujisaka 5 and Tsuyuake backgrounds, respectively. All the $\mathrm{T}_{1}$ lines (70 to 80 individual plants per line) segregated for response to $\mathrm{CH} 43$, and resistance cosegregated with the hygromycin selection gene (Fig. 2A and B). Among these $T_{1}$ lines, 20 lines in Fujisaka 5 background and 15 lines in Tsuyuake background displayed a 3R:1S segregation ratio for their response to $\mathrm{CH} 43$, indicating that most of these transformants are of single T-DNA copy. Similarly, multiple transgenic lines of Nipponbare (which is moderately susceptible to isolate $\mathrm{CH} 43$ ) transformed with $N B S$-2 also showed high resistance (disease score 0) to CH43 (Supplementary Fig. S1). 
Together, these results confirmed that NBS-2 presents the functional Pi64 gene in cv. YMG.

\section{Resistance specificity of transgenic plants.}

To examine the specificity of response in transgenic plants carrying the $N B S-2$ gene, three homozygous $\mathrm{T}_{2}$ transgenic lines and seven reference lines were selected and inoculated with a range of $M$. oryzae isolates (Table 1). We found that all the tested transgenic lines were resistant to six indica-sourced isolates CH43, ZJ14, CH899, CH971, GD02-15-1-1, and FJ0718-1 but were susceptible to one indica-sourced isolate, $\mathrm{CH} 375$, and two japonica-sourced isolates, 99-26-2 and 97-116-2; however, the three Pish-harboring lines Nipponbare, IRBLsh-S, and IRBLsh-B and the Pi37-harboring line St No.1 were susceptible or moderately susceptible or resistant to all these isolates (Table 1). Thus the Pi64 gene exhibited racespecific resistance and seemed to confer a different resistance spectrum from both Pish and Pi37 to these tested isolates

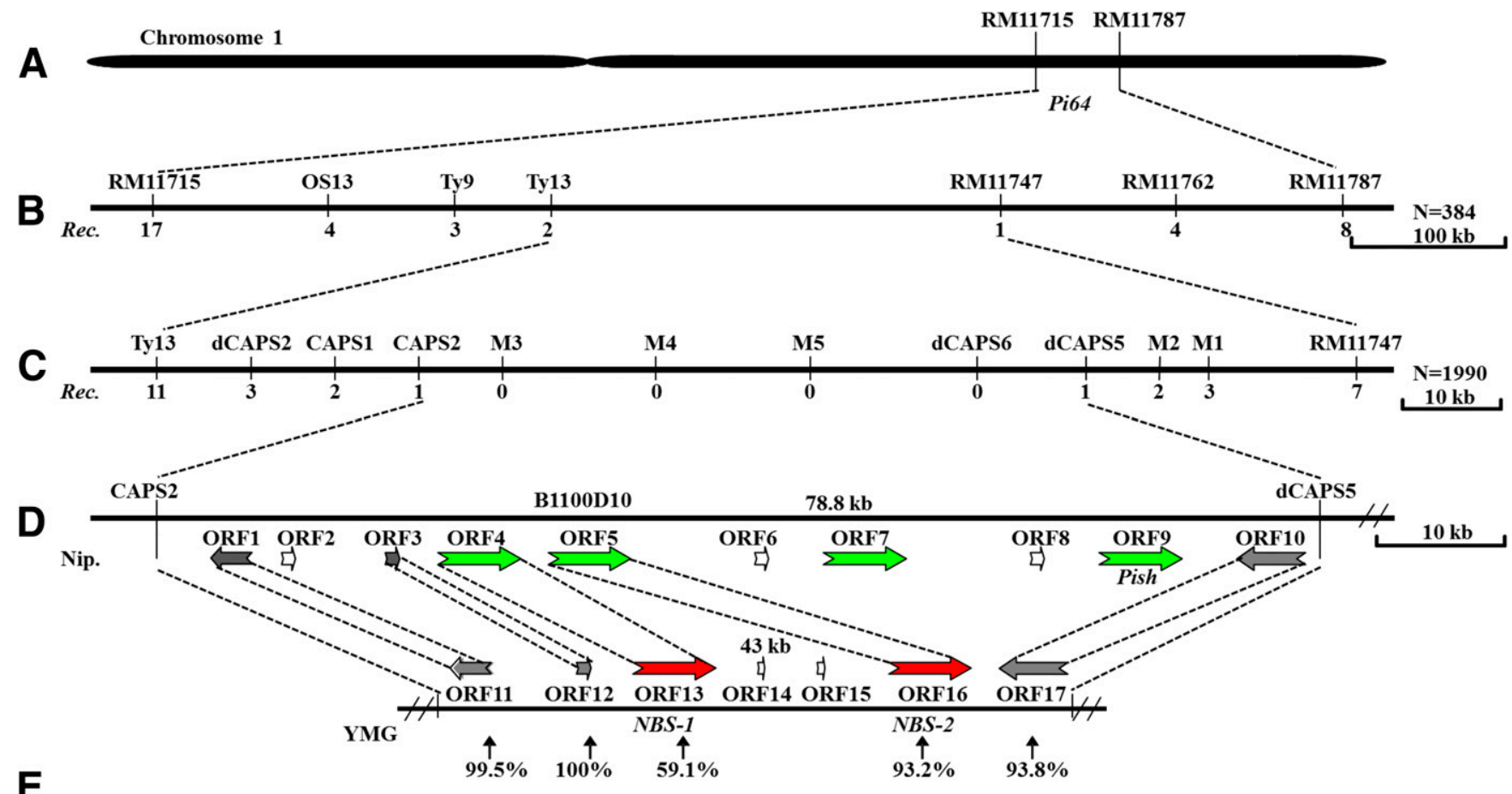

Fig. 1. Genetic and physical maps of the Pi64 gene. A, The Pi64 gene is located on chromosome 1L between simple sequence repeat markers RM11715 and RM11787. B, The Pi64 gene was preliminarily delimited to the Ty13-RM11747 interval using 384 susceptible $\mathrm{F}_{2}$ individuals; marker names and number of recombinants are shown. C, Fine genetic mapping of the Pi64 gene based on 1,990 susceptible $\mathrm{F}_{2}$ individuals. D, Physical map around a $78.8-\mathrm{kb}$ Pi64 region in the reference Nipponbare genome on BAC clone B1100D10 and the corresponding 43-kb region in the japonica landrace Yangmaogu (YMG) genome. Open reading frames (ORF) were predicted in the mapped regions of the Nipponbare and YMG genomes $(n=10$ [ORF1 to 10] and 7 [ORF11 to 17], respectively. Green arrows indicate the four nucleotide-binding site-leucine-rich repeat (NBS-LRR) proteins in the Nipponbare, red arrows indicate two predicted NBS-LRR proteins in the YMG, gray arrows indicate other predicted homologous proteins in Nipponbare and YMG, and blank arrows indicate the predicted nonhomologous proteins. E, Nucleotide identities of the predicted homologous genes in Nipponbare and YMG.

A

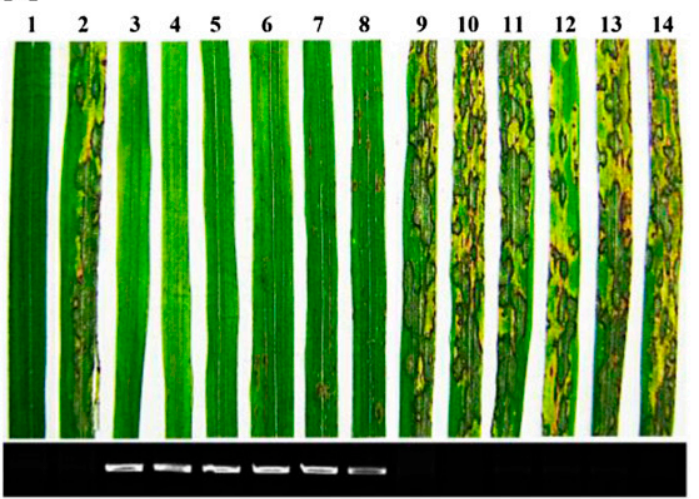

B

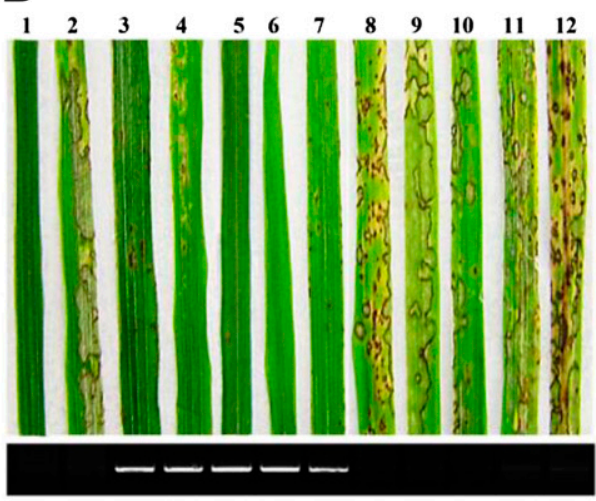

C

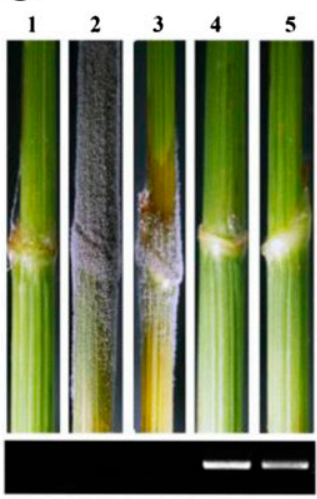

Fig. 2. Complementation test of susceptible rice cultivars with the Pi64 gene using Magnaporthe oryzae isolate CH43 (top) and molecular analysis of transgenic lines using primer Hyg F/R (bottom). A, Numbers 1 and 2 represent rice cultivars Yangmaogu (YMG) and Fujisaka 5, respectively; numbers from 3 to 8 and 9 to 14 represent resistant and susceptible $T_{1}$ progeny, respectively, of one transgenic line (Fujisaka 5-TG) in Fujisaka 5. B, Numbers 1 and 2 represent rice cultivars YMG and Tsuyuake, respectively; numbers from 3 to 7 and 8 to 12 represent resistant and susceptible $\mathrm{T}_{1}$ progeny, respectively, of one transgenic line (Tsuyuake-TG) in Tsuyuake. C, Reaction to $M$. oryzae isolate CH43 in necks of YMG, cv. Lijiangxintuanheigu, Tsuyuake, and two Pi64-transgenic T, plants of the Tsuyuake-TG-3 line (numbers 1 to 5 ). 
(Table 1). We thus concluded that Pi64 is a novel $R$ gene unique to YMG.

To assess the resistance spectrum of Pi64, $\mathrm{T}_{3}$ seedling from the transgenic line, Fujisaka 5-TG-2 (Pi64+Pii+Pik-s) (Table 1), were inoculated with 100 Fujisaka $5(P i i+P i k-s)$-virulent isolates screened from a total of 557 tested isolates. We found that Pi64 could resist $70.21 \%$ of the 47 indica-sourced isolates tested (Supplementary Table S8) and, on the contrary, showed susceptibility to all 53 japonica-sourced isolates. This result indicated that Pi64 could be used as a broad-spectrum resistance $R$ gene for indica rice breeding in China.

Pi64 confers neck blast resistance to isolate CH43.

To date, only two blast $R$ genes, $P i 25$ and $P b l$, have been identified to confer resistance to neck and panicle blast, respectively (Chen et al. 2011; Hayashi et al. 2010; Zhuang et al. 2002). To determine whether Pi64 confers resistance to neck blast, we evaluated various lines, including YMG, LTH, and the transgenic derivatives of Tsuyuake, for response to isolate CH43 using an in vitro inoculation method (Hao et al. 2009, Zhuang et al. 2002). As shown in Figure $2 \mathrm{C}$ and Supplementary Figure S2, YMG showed high neck resistance to CH43 with no lesions, whereas LTH and Tsuyuake were highly susceptible, with lesions of over $20 \mathrm{~mm}$ in length. The homozygous $\mathrm{T}_{2}$ transgenic line Tsuyuake-TG-3 harboring Pi64 displayed a strong resistance to $\mathrm{CH} 43$ as YMG. This indicated that Pi64 conferred resistance to both leaf blast and neck blast.

\section{Structure of the Pi64 gene.}

To obtain full-length cDNA sequences of Pi64 gene, we performed rapid amplification of cDNA ends polymerase chain reaction (RACE-PCR) and reverse transcription (RT)-PCR and sequenced the PCR products. Comparison of full-length cDNA and genomic DNA sequences showed that Pi64 contained a 3,867-bp coding region, a 717-bp 5' untranslated region (UTR), and a 504-bp 3' UTR. One intron of $108 \mathrm{bp}$ was positioned within the $3^{\prime}$ UTR, starting 38 bp downstream of the TGA stop codon (Fig. 3A).

The Pi64 gene is predicted to encode a polypeptide of 1,288 amino acids with a molecular mass of $146 \mathrm{kDa}$. Protein motif analysis showed that the $\mathrm{N}$-terminus of the protein contains two potential CC domains (residues 51 to 88 and 124 to 152 ); the NBS domain contains three typical kinase motifs: GGAGKS (residues 220 to 225, kinase 1a), LLVLDDV (residues 295 to 301, kinase 2), and GSRVLVTSRQ (residues325 to 364, kinase $3 \mathrm{a}$ ); and the C-terminal region contains 27 irregular LRR repeats (residues 590 to 1288) (Fig. 3B) (Martin et al. 2003; McDonnell et al. 2006; van der Biezen and Jones, 1998). Thus, the Pi64 gene encodes a typical CC-NBS-LRR protein. Phylogenetic analysis of Pi64 and other blast $R$ or NBS-LRR type proteins, i.e., Pit, Pish, Pid3, Pib, Pi9, Pi2/Piz-5, Piz-t, Pi36, Pita, Pb1, Pi5-1 and Pi5-2 (Pi5), Pia-1, and Pia-2 (Pia), Pik-1 and Pik-2 (Pik), Pikm1-TS and Pikm2-TS(Pik-m), Pikp-1 and Pikp-2 (Pik-p), Pi1-5 and Pi1-6 (Pi1), NBS1 (YMG), LOC_Os01g57270, LOC_Os01g57280, and LOC_Os01g57310 showed that the 27 proteins could be classified into 11 clades at a $47 \%$ similarity level. Pi64, Pish, NBS-1, LOC_Os01g57270, LOC_Os01g57280, and LOC_Os01g57310 were grouped in the same clade (clade III) but distinct from other reported $\mathrm{R}$ proteins (Supplementary Fig. S3).

\section{Subcellular localization of the Pi64 protein.}

To determine the subcellular localization of Pi64, the fulllength coding sequence of Pi64 was fused to the N-terminus of green fluorescent protein (GFP). When transiently expressed in rice leaf protoplasts, the Pi64-GFP fusion protein, like that of the free GFP, was evenly distributed throughout the rice protoplasts. The nuclear marker RPBF-mCherry and chloroplast autofluorescence indicated that Pi64-GFP protein is also localized in the nucleus but not in the chloroplasts (Fig. 4).

\section{Expression pattern analysis of Pi64.}

Rice blast $R$ genes Pid2, Pi9, Pi2/Piz-5, Piz-t, Pi36, Pi5-2, $P i t a$, and $P i 37$ are constitutively expressed, but others, such as Pib, Pi5-1, Pik-m, and Pik-p, exhibit an induced expression pattern (RoyChowdhury et al. 2012; Wang et al. 1999). To determine whether expression of Pi64 in YMG can be induced in response to challenge by $M$. oryzae, we performed quantitative (q)RT-PCR at eight time points $(0,6,12,24,48,72,96$, and $120 \mathrm{~h}$ postinoculation [hpi]). Pi64 expression was detected both before and after inoculation, indicating that this gene is constitutively expressed. The effects of mock and pathogen treatments were similar, and expression of Pi64 slightly increased at $6 \mathrm{hpi}$ and peaked at $12 \mathrm{hpi}$. After that, expression of pi64 decreased, reaching its lowest level at $48 \mathrm{hpi}$, and then, rebounded thereafter until 120 hpi (Supplementary Fig. S4).

Further, examination of the expression patterns of Pi64 showed that Pi64 was expressed in all development stages and all organs (Fig. 5). The strongest expression was detected at the booting stage (flag leaf) and in young leaf sheathes, and there was relatively weak expression at the seedling stage (second or third leaves) and in old leaf sheaths and panicles. To more

Table 1. Disease reactions of transgenic plants and differential cultivars to nine Magnaporthe oryzae isolates (leaf blast)

\begin{tabular}{|c|c|c|c|c|c|c|c|c|c|c|}
\hline \multirow[b]{2}{*}{ Cultivar $^{\mathbf{a}}$} & \multirow[b]{2}{*}{$\boldsymbol{R}$ Gene } & \multicolumn{9}{|c|}{ Blast isolate } \\
\hline & & CH 43 & $99-26-2$ & ZJ 14 & CH 899 & CH 971 & GD02-15-1-1 & FJ07-18-1 & CH 357 & 97-116-2 \\
\hline \multicolumn{11}{|l|}{ Transgenic lines } \\
\hline Fujisaka 5-TG-2 & Pi64+Pii+Pik-s & $\mathrm{R}^{\mathrm{b}}$ & $\mathrm{S}^{\mathrm{c}}$ & $\mathrm{R}$ & $\mathrm{R}$ & $\mathrm{R}$ & $\mathrm{R}$ & $\mathrm{R}$ & $\mathrm{S}$ & $\mathrm{S}$ \\
\hline Tsuyuake-TG-3 & Pi64+Pik-m & $\mathrm{R}$ & $\mathrm{S}$ & $\mathrm{R}$ & $\mathrm{R}$ & $\mathrm{R}$ & $\mathrm{R}$ & $\mathrm{R}$ & $\mathrm{S}$ & $\mathrm{S}$ \\
\hline Nipponbare-TG-5 & Pi64+Pish & $\mathrm{R}$ & $\mathrm{S}$ & $\mathrm{R}$ & $\mathrm{R}$ & $\mathrm{R}$ & $\mathrm{R}$ & $\mathrm{R}$ & $\mathrm{S}$ & $\mathrm{S}$ \\
\hline \multicolumn{11}{|l|}{ Reference lines } \\
\hline YMG & Pi64+? & $\mathrm{R}$ & $\mathrm{R}$ & $\mathrm{R}$ & $\mathrm{R}$ & $\mathrm{R}$ & $\mathrm{R}$ & $\mathrm{R}$ & $\mathrm{R}$ & $\mathrm{R}$ \\
\hline Fujisaka $5^{*}$ & Pii+Pik-s & $\mathrm{S}$ & $\mathrm{S}$ & $\mathrm{S}$ & $\mathrm{S}$ & $\mathrm{S}$ & $\mathrm{S}$ & $\mathrm{S}$ & $\mathrm{S}$ & $\mathrm{S}$ \\
\hline Tsuyuake* & Pik-m & $\mathrm{S}$ & $\mathrm{S}$ & $\mathrm{R}$ & $\mathrm{R}$ & $\mathrm{S}$ & $\mathrm{S}$ & $\mathrm{R}$ & $\mathrm{S}$ & $\mathrm{S}$ \\
\hline Nipponbare* & Pish & $\mathrm{MS}^{\mathrm{d}}$ & $\mathrm{S}$ & MS & $\mathrm{S}$ & $\mathrm{S}$ & MS & MS & $\mathrm{S}$ & $\mathrm{S}$ \\
\hline St No.1** & $P i 37+P i s h+P b 1$ & MS & $\mathrm{S}$ & $\mathrm{S}$ & MR & $\mathrm{S}$ & MR & MR & MS & MS \\
\hline IRBLsh-S & Pish & MS & $\mathrm{S}$ & MS & MS & $\mathrm{S}$ & $\mathrm{S}$ & MS & $\mathrm{S}$ & $\mathrm{S}$ \\
\hline IRBLsh-B & Pish & MS & $\mathrm{S}$ & $\mathrm{S}$ & $\mathrm{S}$ & $\mathrm{S}$ & $\mathrm{S}$ & MS & $\mathrm{S}$ & $\mathrm{S}$ \\
\hline LTH & None & $\mathrm{S}$ & $\mathrm{S}$ & $\mathrm{S}$ & $\mathrm{S}$ & $\mathrm{S}$ & $\mathrm{S}$ & $\mathrm{S}$ & $\mathrm{S}$ & $\mathrm{S}$ \\
\hline
\end{tabular}


precisely examine the spatial and temporal expression patterns of Pi64, we constructed a pPi64pro::GUS ( $\beta$-glucuronidase) construct and transformed it into cv. YMG. Consistent with the qRT-PCR results, we observed GUS signals in various tissues of transgenic plants, including roots, culms, leaf sheaths, leaf blades, and various reproductive organs (Supplementary Fig. S5). These results indicated that Pi64 is broadly expressed at all developmental stages and in all organs examined.

\section{Development of allele-specific markers for Pi64.}

Genomic sequence comparison showed the region from the TGA downstream of the $N B S-1$ allele to the ATG upstream of the Pi64 allele in YMG shared hardly any similarity to the corresponding regions in Nipponbare (DNA sequence similarity $14.6 \%$, Gaps $81.2 \%$ ) (Supplementary Data File S2) and 93-11 (DNA sequence similarity 19.8\%, Gaps 77.6\%) (Supplementary Data File S3) and a TIR-like DNA transposon, with 11-bp terminal inverted repeats bordered by 8-bp target site duplications (ATTGGAAG) (Wicker et al. 2007), was only detected in YMG, located 314 bp upstream of the ATG start codon of Pi64 (Fig. 6A). We designed three sets of primers,
YRT4, YRT5, and YRT6 in the ATG upstream region of Pi64 and Pish (Fig. 6A) and used them to genotype six rice landraces originated from the same Yunnan plateau region as YMG, seven typical japonica, and three typical indica cultivars (Fig. 6B). As shown in Figure 6B, a 421-bp fragment was amplified by YRT4 from YMG only, a 1,391-bp fragment from YMG and a 633-bp fragment by YRT5 from the other 10 cultivars, and a 1,016-bp fragment by YRT6 from the remaining six cultivars. The 17 cultivars could be classified into three genotypes based on the presence or absence of amplified fragments by these primer sets, i.e., the YMG type contains YMG only, the 93-11 type contains 10 cultivars, and the Nipponbare type contains six cultivars (Fig. 6B; Supplementary Table S9). Genotyping analysis of an additional 58 Chinese leading rice cultivars (Shi et al. 2009) and 199 accessions of the mini-core collection of Chinese germplasm (Yang et al. 2009) showed that all of them were either 93-11 or Nipponbare type. Thus, primers YRT4 and YRT5 can be used as allele-specific markers for Pi64.

To further evaluate the practicability of YRT4, YRT5, and YRT6 in marker-assisted selection (MAS), two populations, one containing $97 \mathrm{~F}_{2}$ progenies derived from a cross between

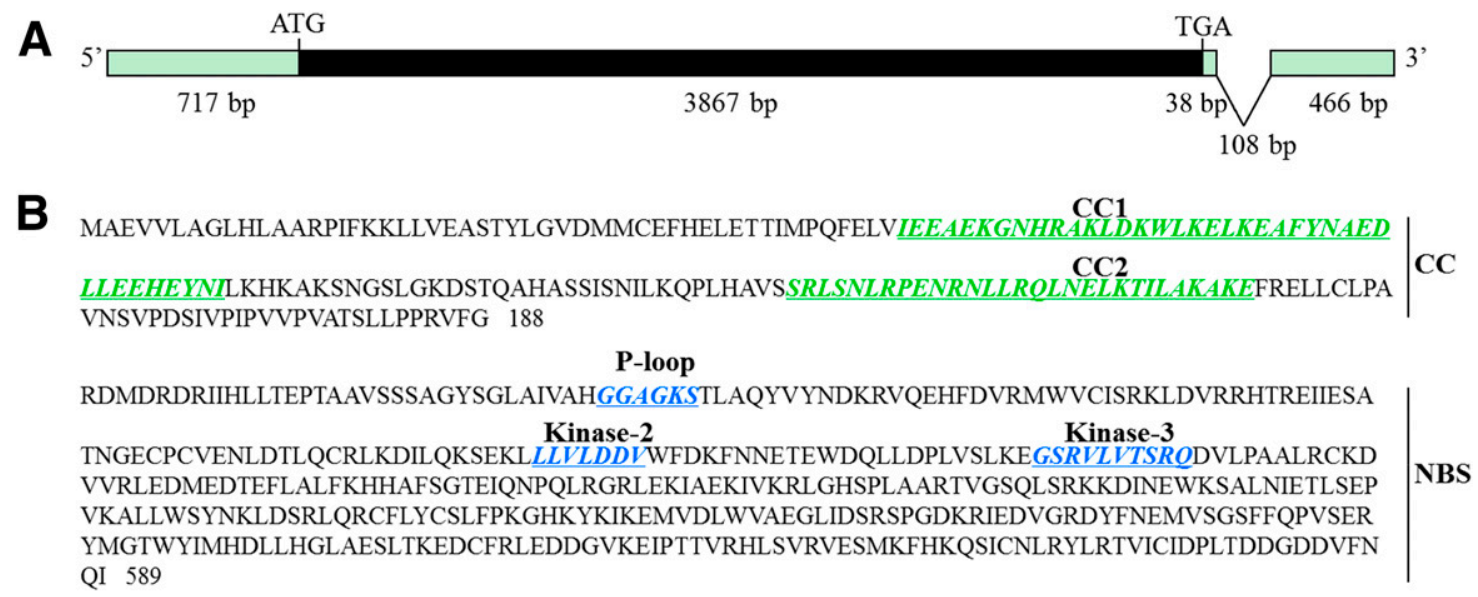

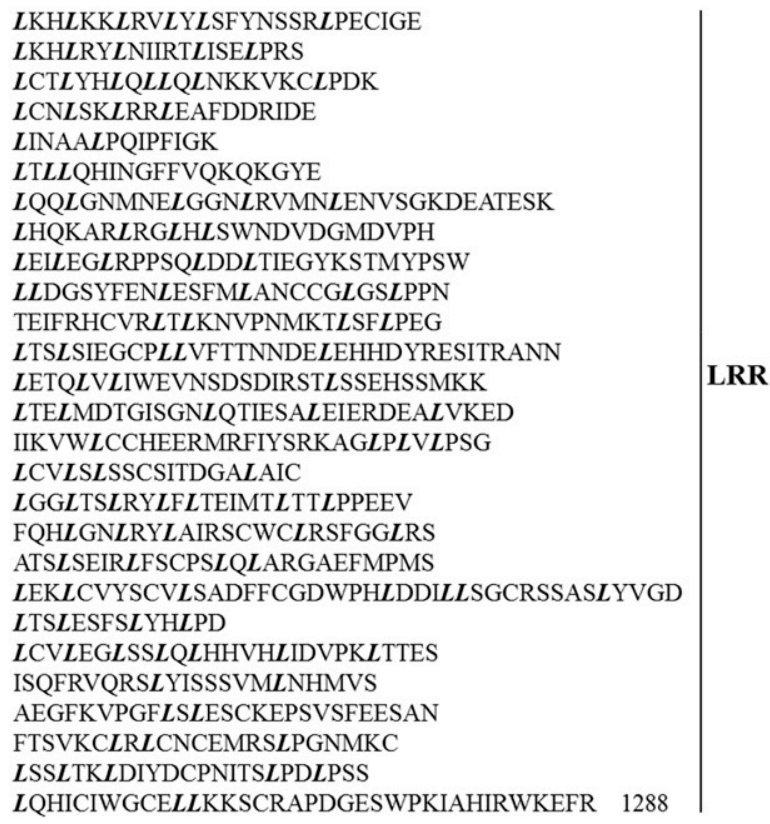

Fig. 3. Structure of Pi64 and its deduced amino acid sequence. A, Gene structure of Pi64. ATG and TGA indicate the translation start and stop codon, respectively. The black box represents exon, lines denote introns, and blank boxes indicate $5^{\prime}$ and $3^{\prime}$ untranslated regions. B, Deduced peptide sequence of the Pi64 gene product. The two coiled-coil (CC) motifs are shown in green. The three conserved motifs (P-loop, kinase-2, kinase-3) of the nucleotide-binding site (NBS) region are shown in blue. The C-terminal leucine-rich repeat (LRR) region is shown detached from the rest of the sequence and contains 27 imperfect repeat LRR units. 
YMG and CO39 (93-11 type) and the other containing 47 $\mathrm{BC}_{5} \mathrm{~F}_{2}$ progenies derived from a backcross YMG and LTH (recurrent parent, Nipponbare type), were genotyped using these three markers and were phenotyped by inoculation with two differential blast isolates, $\mathrm{CH} 899$ and $\mathrm{CH} 43$, respectively (the $F_{2}$ genotypes were confirmed by their $F_{3}$ families). As shown in Figure 7 and Supplementary Table S10, YRT4 could precisely monitor the Pi64 gene but it could not differentiate the Pi64Pi64 and Pi64pi64 genotypes in either of the populations; whereas YRT5 could precisely distinguish Pi64Pi64 from Pi64pi64 in the population of YMG $\times$ CO39. The Pi64Pi64 and Pi64pi64 genotypes could also be distinguished using the YRT4 + YRT6 or YRT5 + YRT6 combinations in the population of $(\mathrm{YMG} \times \mathrm{LTH}) \times \mathrm{LTH}($ Fig. 7$)$. Thus, YRT4 was a dominant Pi64-specific marker, whereas YRT5 alone and in combination with YRT6 could be used as codominant Pi64specific markers for MAS in populations derived from crosses between YMG and 93-11-type cultivars and between YMG and Nipponbare-type cultivars, respectively.

\section{DISCUSSION}

The utilization of host $R$ genes is considered to be the most effective and economic means to control blast in rice. In recent years, many plant pathologists and breeders have evaluated blast-resistant germplasm resources and developed several broad-spectrum or durably resistant cultivars (Huang et al. 2011; Tan et al. 2000; Wu et al. 2004). However, blast $R$ genes were only analyzed in a few BSR or durable rice cultivars (e.g., Moroberekan, Suweon 365, Sanhuangzhan 2, Digu, and Gumei 2)
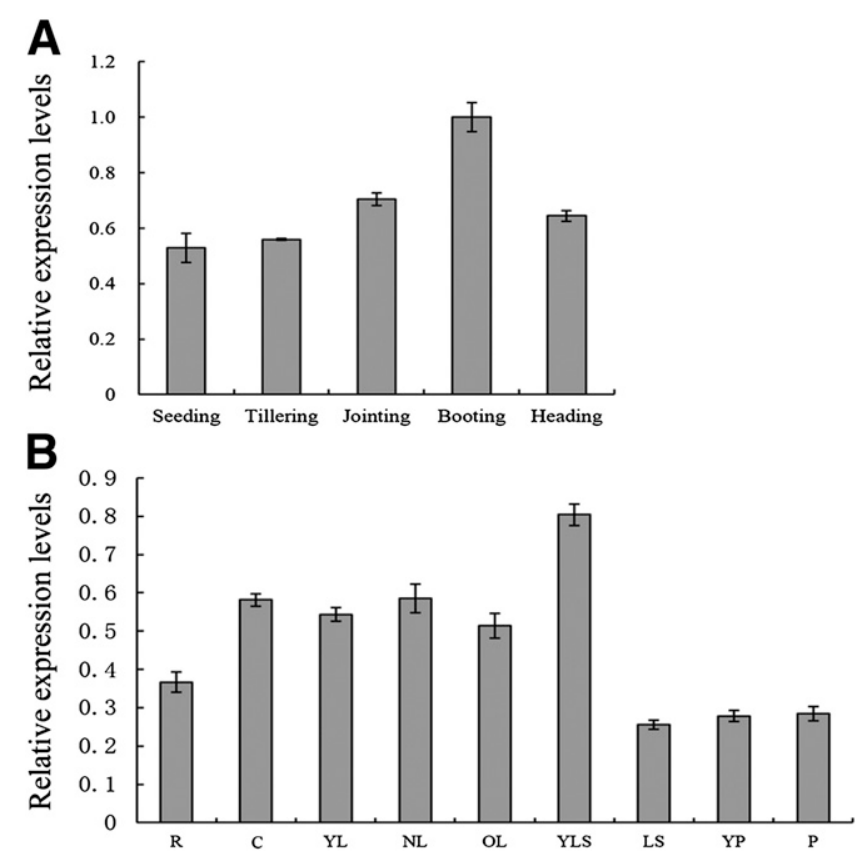

Fig. 5. Expression pattern of Pi64 in different developmental stages and various organs in japonica landrace Yangmaogu (YMG). A, Quantitative reverse transcription-polymerase chain reaction (qRT-PCR) analysis of Pi64 expression in different developmental stages. B, qRT-PCR analysis of Pi64 expression in roots (R), culms (C), 5- to 7-cm unexpanded young leaves (YL), new leaves (NL), old leaves (OL), young leave sheaths (YLS), leaf sheaths (LS), young panicles (YP), and panicles (P), respectively. Values are means \pm standard deviation of three biological repeats.
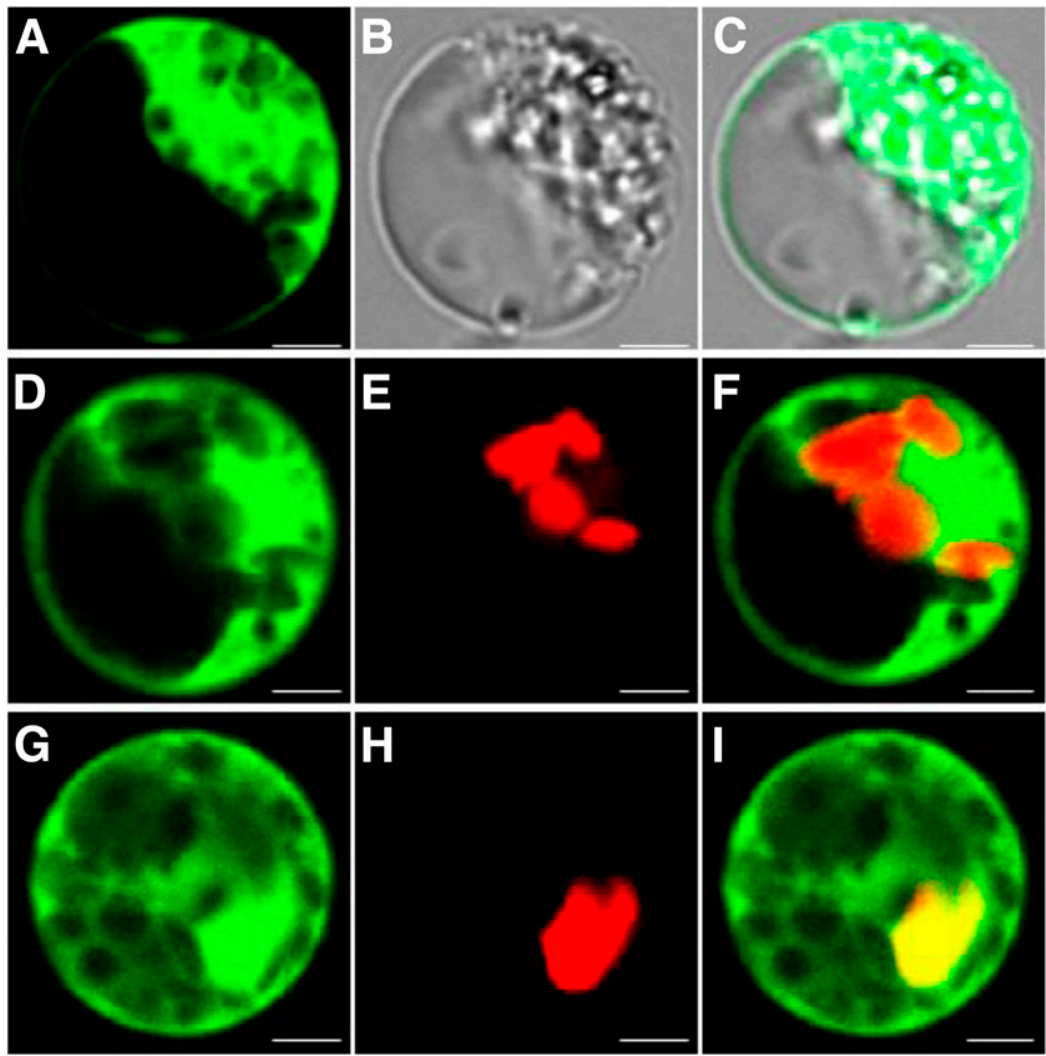

Fig. 4. Subcelluar localization of Pi64 protein in rice protoplasts. Green fluorescence shows green fluorescent protein (GFP), red fluorescence shows chloroplast autofluorescence, and yellow fluorescence shows the emerged images. A, Free GFP is detected in both the nucleus and cytoplasm. B, Bright field image. C, A and B merged. D and G, Pi64-GFP fusion protein is detected in the nucleus and cytoplasm. E, Chloroplast autofluorenscence. F, D and E merged. H, Nuclear localization of RPBF-mCherry (a nuclear marker). I, G and H merged. Scale bars $=5 \mu \mathrm{m}$. 
and have been rarely utilized in rice breeding so far (Lei et al. 2013; Liu et al. 2013; Wu et al. 2007). Thus, identification and isolation of novel $R$ genes are still urgently needed for breeding durable and broad-spectrum blast resistance rice cultivars. In this work, we showed that $\mathrm{cv}$. YMG is resistant to all tested blast isolates (total 557) collected from a wide range of rice-cropping regions in China. Our genetic analysis suggested that it contains at least three major dominant $R$ genes. Using a map-based cloning approach, we cloned the Pi64 gene, which confers both leaf and neck resistance to the blast isolate $\mathrm{CH} 43$ in a dominant fashion. We show that Pi64 encodes a novel CC-NBS-LRR R protein that is localized to both the cytoplasm and nucleus. Consistent with its leaf and neck blast resistance, expression of Pi64 is constitutive in different developmental stages and tissues.
$N B S$ - LRR genes represent the largest class of $R$ genes in plants and tend to be clustered in closely related gene groups (Martin et al. 2003; Richter and Ronald 2000), such as the rice Pik and Pi9 loci (Zhai et al. 2011; Zhou et al. 2007). In the present study, Pi64 was delimited to a 43-kb genomic region in YMG, which corresponds to a 78.8-kb interval in Nipponbare. Interestingly, four $N B S$ - $L R R$ genes, arranged as tandem repeats (LOC_OsO1g57270, LOC_Os01g57280, LOC_Os01g57310, and $L O C \_O s 01 g 57340$ ) were identified in the Nipponbare interval (Fig. 1). Among these NBS-LRR genes, LOC_Os01g57340 was previously identified as Pish, whereas LOC_OsO1g57310 (Npi37-3) is reported to be an allele of the $R$ gene Pi37 (Lin et al. 2007; Takahashi et al. 2010). These four NBS-LRR genes all belong to the $R p l R$ gene family and may have arisen from two

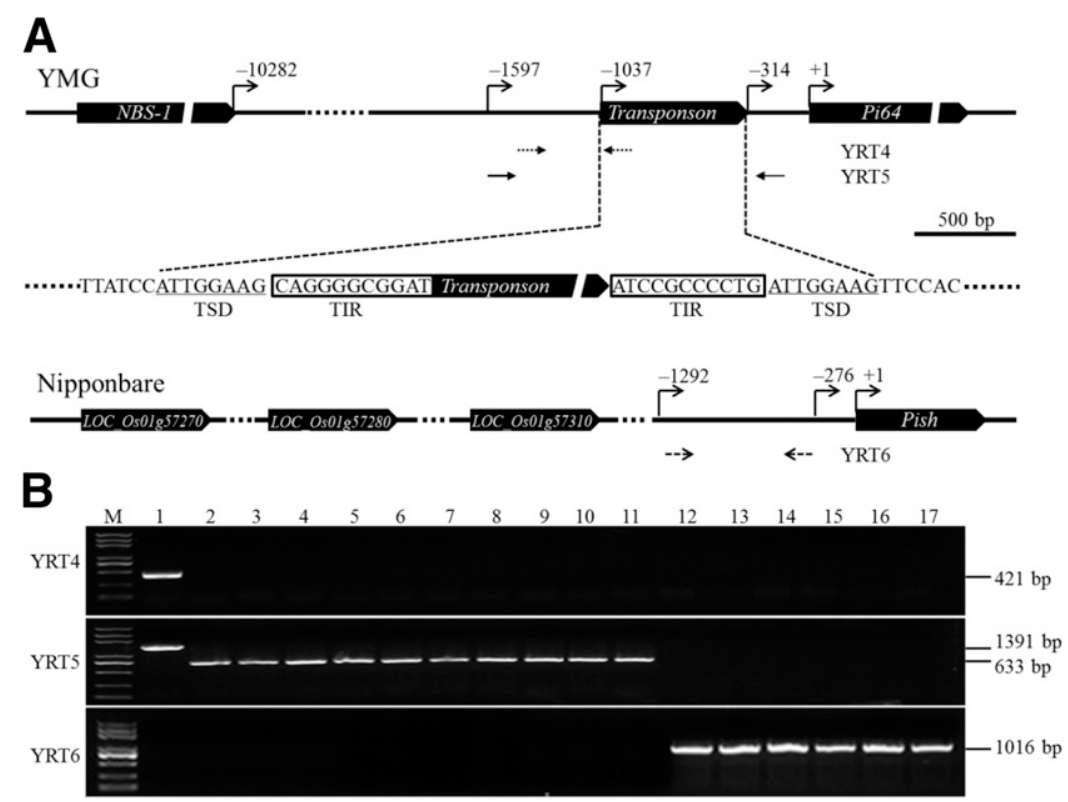

Fig. 6. Development of Pi64-specific markers and genotype analysis of rice cultivars. A, A schematic representation of the sequence from the TGA downstream of $N B S-1$ to the ATG upstream of Pi64 in cv. Yangmaogu (YMG). B, Polymerase chain reaction products amplified from genomic DNA prepared from 17 rice cultivars, using primers YRT4, YRT5, and YRT6. ATG indicates the translation start site and TGA indicates the termination site. TSD, target site duplication; TIR, terminal inverted repeat. 1 to 17: cvs. YMG, 93-11, Fujisaka 5, Tsuyuake, K59, Qitoubaigu, Momi, Kinmaze, Aichi Asahi, CO39, Ningjing 11, Nipponbare, Huangpinuo, Laozaogu, Ximaxian, Dongnong 363, and Lijiangxintuanheigu, respectively.

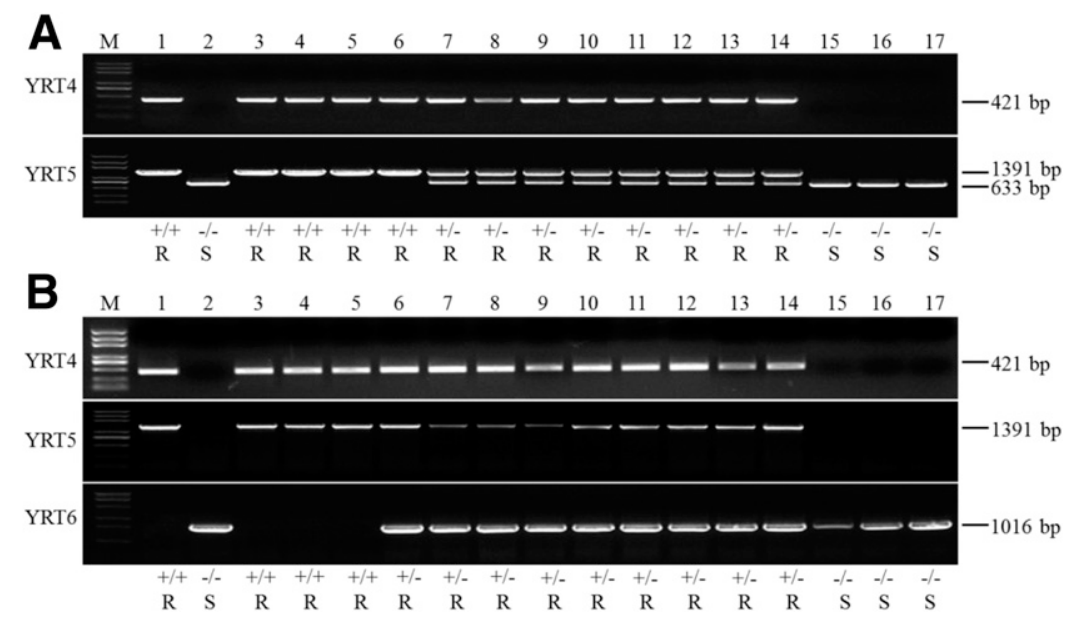

Fig. 7. Detection of the Pi64 gene in progenies derived from the crosses between cvs. Yangmaogu (YMG) and CO39 or between cvs. YMG and Lijiangxintuanheigu $(\mathrm{LTH})$. A, Polymerase chain reaction (PCR) products of genomic DNA prepared from two parents (1, YMG and 2, CO39) and their $15 \mathrm{~F}_{2}$ progenies ( 3 to 17 ) amplified by primers YRT4 and YRT5. B, PCR products of genomic DNA prepared from two parents $(1, \mathrm{YMG}$ and $2, \mathrm{LTH})$ and their $15 \mathrm{BC}_{5} \mathrm{~F}_{2}$ progenies (3 to 17) amplified by primers YRT4, YRT5 and YRT6. M, marker; +/+, Pi64/Pi64; +/-, Pi64/pi64; -/-, pi64/pi64; R, resistant; and S, susceptible. 
duplication events of an ancestral NBS-LRR gene (Takahashi et al. 2010; Luo et al. 2011). Further, it has been postulated that the difference in 19 residues in the LRR domain between Pish and LOC_OsOlg57310 are important for maintaining the target recognition site of Pish.

Despite the presence of Pish and LOC_Os01g57310 (Npi37-3), cv. Nipponbare shows moderate susceptibility to $M$. oryzae isolates containing a putative Pi64-avirulent gene (Table 1), indicating that none of the four NBS-LRR genes confer resistance to the Pi64-avirulent isolates. Comparison of YMG and Nipponbare sequences in this region suggested that a $35.8-\mathrm{kb}$ deletion in YMG causes the loss of LOC_OsO1g57310 and Pish and several other small ORF (Fig. 1). As a result, YMG contains only two genes encoding NBS-LRR proteins (NBS-1 and NBS-2), and they share a modest sequence similarity to each other (approximately $50 \%$ ) and highest nucleotide similarity to LOC_OsOlg57270 (59.1\%) and LOC_OsO1g57280 (93.20\%), respectively (Fig. 1). This indicated that $N B S-1$ and $N B S-2$ could be allelic to LOC_OsO1g57270 and LOC_OsO1g57280, respectively. We further showed that transformants containing NBS-1 are all susceptible to $\mathrm{CH} 43$, while all transformants of $N B S-2$ are resistant to CH43, suggesting that NBS-2 corresponds to Pi64 (Fig. 2). Comparison of the NBS domains of Pi64 and Pish revealed that there is only one amino acid residue substitution (Q364R) corresponding to kinase 3a (Supplementary Fig. S6). As previously reported, the kinase $3 \mathrm{a}$ motif is involved in binding purine or ribose and contains a conserved tyrosine $(\mathrm{Y})$ or arginine $(\mathrm{R})$ residue (Traut 1994), and thus, the single amino acid difference in kinase 3a may underlie the functional differences between Pi64 and Pish. In addition, the LRR region of Pi64 containing 27 irregular LRR, which shared $91.58 \%$ sequence identity with the 29 irregular LRR of Pish. The slight difference in LRR domains between Pi64 and Pish may also be involved in specific recognition of corresponding avirulence genes. Further characterization of the other NBS-LRR genes in this cluster may lead to the identification of new $R$ genes and a better understanding of the differentiation in resistance specificity at the molecular level and may also shed light on the evolution of new $R$ genes.

The genetic analysis showed YMG contains at least three $R$ genes, depending upon the particular $M$. oryzae isolate, that is to say, the BSR phenotype of YMG is likely due to a combination of multiple $R$ genes or even quantitative trait loci (QTL). To successfully utilize this elite resistant resource in future rice breeding programs, we have been trying to fine-map and clone each of the $R$ genes or QTL in YMG and determine their resistance spectrum and utilization strategy for blast resistance breeding. By now, we have successfully cloned the Pi64 gene and fine-mapped another blast $R$ gene, $P i 65(t)$, to an approximately $600-\mathrm{kb}$ interval on the short arm of chromosome 4 . As far as it is concerned, the Pi64 gene in transgenic plants showed strong specificity to $M$. oryzae isolates, which conferred highlevel resistance to about $70 \%$ of indica-sourced isolates, but was completely susceptible to almost all japonica-sourced isolates. Even so, we think that Pi64 can be applied as a BSR gene alone or in combination with other $R$ genes with complementary resistance spectrums to local $M$. oryzae isolates in the indica ricecropping regions of China. In addition, we confirmed that Pi64 could also confer resistance to neck blast, using an in vitro inoculation method (Fig. 2C) (Hao et al. 2009, Zhuang et al. 2002). The in vitro inoculation, which has been studied and applied in rice neck (panicle) blast assays in China since the early 1990s, could effectively differentiate the resistance of different rice cultivars and also well reflect the panicle blast resistance of the whole rice plants (Chai and Jin 1995; Hao et al. 2009, 2012; Hu et al. 2006; Li et al. 2010; Rao et al. 2005; Sun and Su 1992; Sun et al. 1993; Wu et al. 2000; Zhuang et al. 2002).
It is not difficult to understand that Pi64 can confer resistance to both leaf and neck blast in rice due to its high level and constitutive expression at all developmental stages and in all tissues examined, particularly at the booting stage and in young leaf tissues (Fig. 5). This observed pattern of constitutive expression seems to offer some leads, e.g., timely and strong expression of some NBS-LRR genes are important for the neck or panicle blast, that still need to be validated by testing expression patterns and neck resistance of some other constitutive expression $R$ genes, such as Pita, Pi9, Pi2 and Piz-t. In conclusion, the Pi64 gene can provide high-level and stable leaf and neck blast resistance to most of the Chinese indica-sourced M. oryzae isolates and this specificity of resistance of Pi64 make it a good resource for application in indica rice breeding. We also developed multiple allele-specific markers of Pi64 (Figs. 6 and 7) that should facilitate breeding of new rice cultivars harboring Pi64 through either MAS or $R$-gene pyramiding. Considering that the broad-spectrum resistance of cv. YMG to both indica- and japonica-sourced M. oryzae isolates, we need to accelerate identification of the other $R$ genes or QTL present in this cultivar in order to comprehensively dissect its resistance mechanism and to provide useful information for utilizing this elite blast-resistant resource in rice breeding.

\section{MATERIALS AND METHODS}

Plant materials and growth conditions.

A total of 281 rice cultivars and lines were used in this study. Among them, YMG and universally susceptible cv. LTH and seven ML, IRBL9-W, IRBLz5-CA, IRBLz-Fu, IRBL1-CL, IRBLb-B, IRBLzt-T, and IRBLta-K1, were evaluated for blast response. Cultivars Fujisaka 5, Tsuyuake, Nipponbare (Pish donor), and the two ML IRBLsh-S and IRBLsh-B (Table 1) were used as receptor genotypes for Pi64 transformation or for assays of $R$-gene specificity with $M$. oryzae isolates, and 274 cultivars were used for a genotypic assay of three Pi64-specific markers. YMG (female parent) was crossed and backcrossed to LTH (male and recurrent parent) to develop $F_{1}, F_{2}, F_{3}$, and $\mathrm{BC}_{1} \mathrm{~F}_{1}$ progenies for genetic analysis and gene mapping. Additionally, the $\mathrm{F}_{2}$ population was derived from a cross between YMG and $\mathrm{CO} 39$ and one $\mathrm{BC}_{5} \mathrm{~F}_{2}$ population was derived from a cross between YMG and LTH (recurrent parent). The seven ML were provided by Y. Fukuta, The International Rice Research Institute, Los Baños, The Philippines. The cv. St No.1 was provided by L. Zhu, Institute of Genetics and Developmental Biology, Chinese Academy of Sciences, Beijing. The other cultivars and lines were maintained at the Institute of Crop Science, Chinese Academy of Agricultural Sciences (CAAS), Beijing.

Seedlings were grown in $60 \times 30 \times 5-\mathrm{cm}$, watered and fertilized plastic seedling trays in a greenhouse as described by Li et al. (2008). Briefly, moistened seeds were sown in rows together with the two parents. For blast tests, 15 seeds of each reference line (Table 1) and the two parents were sown in rows (15 seed per row) in duplicated trays. For genetic analysis and gene mapping, about 300 seeds of the $\mathrm{F}_{2}, 50$ seeds of the $\mathrm{BC}_{1} \mathrm{~F}_{1}$ population, or 20 seeds of each $\mathrm{F}_{3}$ family together with 15 seeds of each parent were sown in rows in trays.

\section{M. oryzae isolates and disease evaluation.}

A total of $557 \mathrm{M}$. oryzae isolates collected from different rice-growing regions of China were evaluated for virulence on YMG. Among them, 11 isolates-seven indica-sourced isolates, i.e., CH43 (pathotype 426.4), ZJ14 (pathotype 006.4), GD02-15-1-1 (pathotype 010.5), FJ07-18-1 (pathotype 007.4), CH357 (pathotype 006.0), CH899 (pathotype 047.6), and CH971 (pathotype 005.4), 
and four japonica-sourced isolates, i.e., 1813-2 (pathotype 001.1), 99-26-2 (pathotype 437.1) and HLJ07-1-1 (pathotype 003.4), and isolate 97-116-2 (pathotype 707.0)_gave clear-cut resistant or susceptible reactions on the 31 monogenic lines (Fukuta et al. 2004). These eleven isolates were selected for use in the analysis of resistance specificity of Pi64-trangenic plants. In addition, 100 isolates that could infect cv. Fujisaka $5(P i i+P i k-s)$ were selected for use in the analysis of resistance spectrum of Pi64. All isolates were stored at the Institute of Crop Science, CAAS.

For evaluation of leaf blast response, inoculum preparation and seedling inoculation followed the procedure described by $\mathrm{Li}$ et al. (2008). Disease responses were scored 6 to 7 days later as described by Kobayashi et al. (2007) and were categorized into four reaction classes, wherein scores 0 and 1 were resistant, score 3- moderately resistant, score $3+$ moderately susceptible, and scores 5, 7, and 9 were considered susceptible. Some seedlings were also transplanted to the field and injectioninoculated, following methods described by Lei et al. (1996) to confirm disease reactions after spray-inoculation.

For evaluation of neck blast resistance, an in vitro inoculation technique (Hao et al. 2009; Zhuang et al. 2002) was used with some modifications. Briefly, 6-cm-long necks containing one to three rachis nodes were cut from panicles and put onto filter paper in petri dishes (10 $\mathrm{cm}$ diameter). The filter paper was presoaked in benzimidazole solution. Both ends of the neck were covered with absorbent cotton that was also presoaked in benzimidazole solution. The nodes were inoculated, using a micropipette, with $2 \mathrm{ml}$ of conidial suspension containing $2 \%$ carboxymethyl cellulose. For each rice cultivar or line, five necks were inoculated. The inoculated necks were incubated in a chamber at $26^{\circ} \mathrm{C}$ for $24 \mathrm{~h}$ in the dark, and then, under a 12-h-light and 12-h-dark cycle. The disease reaction was scored 10 days after inoculation as described by $\mathrm{Li}$ et al. (2010), i.e., resistant, no neck lesion or lesion length $\leq 5 \mathrm{~mm}$ but with no sporulation; moderately resistant, lesion length 5 to $15 \mathrm{~mm}$; and susceptible, lesion length $>15 \mathrm{~mm}$.

\section{DNA extraction and molecular marker development.}

Plant genomic DNA was extracted from frozen young leaves using the CTAB method (Murray and Thompson 1980), and PCR amplifications were performed as described by Li et al. (2008). SSR markers used in this study were adopted from McCouch et al. (2002). InDel markers were developed based on the polymorphisms of available genomic sequences of rice subspecies japonica cv. Nipponbare (International Rice Genome Sequencing Project) and indica cv. 93-11 (Beijing Genomics Institute) using the online software Primer Designing Tool (National Center for Biotechnology Information [NCBI] database). CAPS and dCAPS markers were developed according to sequences from YMG and LTH using dCAPS Finder 2.0.

\section{Linkage analysis and physical map construction.}

A total of 290 SSR and 212 InDel markers, evenly distributed on all 12 chromosomes, were screened for polymorphisms between the parents (Lei et al. 2013; McCouch et al. 2002). The polymorphic markers were then subjected to a modified bulked segregate analysis combined with recessive class analysis (Michelmore et al. 1991). DNA of the two parents, 20 highly susceptible (leaf blast score 7 to 9) and 15 resistant (leaf blast score 0$) F_{2}$ homozygous individuals confirmed by $F_{3}$ progeny tests were used for linkage analysis and location of the $R$ locus. To fine-map the $R$ gene, 384 highly susceptible $\mathrm{F}_{2}$ plants were used for preliminary mapping and an additional 1,516 susceptible $\mathrm{F}_{2}$ individuals were added for fine mapping. The physical map of the target locus was constructed based on the Nipponbare reference sequence (International Rice Genome Sequencing Project 2005) (Rice Genome Annotation Project database).

\section{DNA sequencing and sequence annotation.}

The chromosomal region containing the Pi64 locus in YMG was sequenced by PCR and chromosome walking, based on the reference sequences of Nipponbare and 93-11. To identify candidate $R$ genes in this region, sequence similarity searches were performed with the BLASTN search program (NCBI BLAST database). The genomic sequence was analyzed using the programs RiceGAAS (International Rice Genome Sequencing Project database) and FGENESH (Softberry website) to find putative ORF. The protein motifs were analyzed using the motif scan programs (Massachusetts Institute of Technology Scansite database and the EMBL SMART tool) and the Paircoil2 program. The protein molecular weight was computed by Compute $\mathrm{pI} / \mathrm{MW}$ online (ExPASy Bioinformatics Resource portal). Multiple sequence alignments and a phylogenetic tree were generated by the neighbor-joining method with 1,000 replicates (MEGA Version 4) (Tamura et al. 2007).

\section{Vector construction for complementation analysis.}

Two putative NBS-LRR genes, tentatively named NBS-1 and $N B S-2$, were predicted from the YMG genomic sequence as the candidate genes for Pi64 within the Pi64 locus. To construct a complementary vector carrying NBS-1, highfidelity DNA polymerase, KOD FX (Toyobo), was used to amplify a 8.4-kb EcoRI genomic DNA fragment from YMG with the primer pairs of NBS-1-1305F and NBS-1-1305R. This fragment contained the NBS-1 ATG upstream sequence $(2.6 \mathrm{~kb})$, the whole $N B S-1$ coding region $(3.1 \mathrm{~kb})$ and a TGA downstream sequence $(2.7 \mathrm{~kb})$. The amplified product was inserted into the binary vector pCAMBIA1305 (Cambia), using the Clonetech In-fusion PCR cloning system (TaKaRa), and was named p1305-NBS-1. The NBS-2 gene was constructed into the same binary vector, following the same method and using primer pair NBS-2-1305F and NBS-2$1305 \mathrm{R}$, resulting in a clone of an approximately 7-kb EcoRI fragment from YMG, which was named p1305-NBS-2. This clone contains the NBS-2 ATG upstream sequence $(1.6 \mathrm{~kb}$ ), the whole $N B S-2$ coding region $(3.8 \mathrm{~kb})$, and a TGA downstream sequence $(1.5 \mathrm{~kb})$.

\section{Promoter-GUS and histochemical analysis of GUS activity.}

To study the tissue specificity of the Pi64-promoter, a 1,276bp EcoRI fragment of the Pi64 gene upstream sequence $(-1,597$ to -321) was amplified with primer pair NBS2-ProF and NBS2ProR. The PCR product was integrated upstream of the GUS coding sequence in pCAMBIA1305, in which the Cauliflower mosaic virus (CaMV) 35S promoter was deleted with the Clonetech In-fusion PCR Cloning system (TaKaRa), resulting in pPi64pro::GUS. Histochemical analysis of GUS activity was carried out according to the method described by Inoue et al. (2003).

\section{Rice transformation.}

Binary plasmids p1305-NBS-1and p1305-NBS-2 were introduced into Agrobacterium tumefaciens EHA105 by electroporation (GenePulser Xcell; Bio-Rad), and were transformed into rice cv. Fujisaka 5; Tsuyuake and Nipponbare, which are susceptible to isolate $\mathrm{CH} 43$, and the pPi64pro::GUS vectors were introduced into cv. YMG (Hiei et al. 1994; Qu et al. 2003; Toki et al. 2006). Transgenic plants were confirmed by PCR, using HygF and HygR primers. The PCR products were separated in $1 \%$ agarose gels. $\mathrm{T}_{0}$ and $\mathrm{T}_{1}$ plants were selected and tested for response to leaf and neck blast.

\section{Subcellular localization of Pi64.}

To determine subcellular localization of the Pi64 gene, a 3.87-kb SpeI-BamHI fragment of gene Pi64 was amplified 
from YMG with primers Pi64-GFP-F (SpeI) and Pi64-GFP-R (BamHI). This fragment was cloned into the $\mathrm{N}$-terminus of the GFP coding region in the pAN580 vector, to generate a Pi64GFP fusion expression vector under control of the CaMV 35S promoter using the Clonetech In-fusion PCR cloning system (TaKaRa). After confirmation by sequencing, the chimeric fusion constructs were cotransfected into rice protoplasts as described by Chiu et al. (1996) and Mao et al. (2012). Rice protoplasts were separated from leaves, were incubated in darkness for $16 \mathrm{~h}$ at $28^{\circ} \mathrm{C}$, and were observed by a Nikon Eclipse TE2000-U microscope (Nikon Co.).

\section{Gene expression and full-length cDNA analysis.}

To examine changes in transcript accumulation in response to pathogen infection, three-week-old seedlings of YMG were inoculated with $M$. oryzae isolate $\mathrm{CH} 43$ and a mock control. The leaves were harvested at $0,12,24,48,72,96$, and 120 hpi. Total RNA was prepared from the rice tissue, using an RNAprep pure plant kit (Tiangen), and was treated with RNasefree DNaseI (TaKaRa) to remove contaminating genomic DNA. First-strand cDNA was synthesized using a SuperScript II kit (TaKaRa) according to the manufacturer's instructions. Expression levels of genes NBS-1 and Pi64 were detected by qRT-PCR, with the ubiqutin gene LOC_OsO3g10170 being used as an internal control. The gene expression levels were determined by the $2^{-\Delta \Delta \mathrm{CT}}$ method (Livak and Schmittgen 2001), using a SYBR premix Ex Taq kit (TaKaRa) according to the manufacturer's recommended protocols (Mao et al. 2012).

To obtain the full-length cDNA of Pi64, RACE-PCR with a $5^{\prime}$ full-length RACE kit and 3' full-length RACE Core Set Ver. 2.0 was performed according to the manufacturer's instructions (TaKaRa Biotech Co., Ltd.). Total RNA was isolated from YMG leaves. For 5' end RACE, first-strand cDNA was synthesized as a template, and then, the first round of PCR was performed using 5' RACE outer primer and the gene-specific primer Pi64-1. The resulting PCR product was diluted 50-fold and was used as template for a second round of amplification with 5' RACE inner primer and the gene-specific primer Pi64-2. For the $3^{\prime}$ end RACE, PCR was performed with the gene-specific primers Pi64-3 and Pi64-4 together with the primers 3' RACE outer primer and 3' RACE inner primer, respectively. The sequence between $5^{\prime}$ and $3^{\prime}$ ends was obtained through RT-PCR by using two pairs of gene-specific primers, Pi64cds-F and Pi64cds-R. The RACE ends and intermediate RT-PCR products were cloned into the pEASY-blunt vector (TransGen Biotech) for sequencing.

\section{ACKNOWLEDGMENTS}

We sincerely thank J. Wu at China National Rice Research Institute, and C. Wu at Ceres Inc. for helpful discussion. We also sincerely thank Y. Fukuta at Japan International Research Center for Agricultural Sciences, $X$. Wei at China National Rice Research Institute, and L. Han at Institute of Crop Science, CAAS for kindly providing monogenic lines for blast resistance and Chinese rice germplasm, respectively. We also thank N. Hayashi, National Institute of Agrobiological Sciences, for providing the DNA samples of St No.1 (JP number 10271) for validation of the presence of Pish and $\mathrm{Pbl}$. This work was supported by grants from the National Natural Science Foundation of China (grant number 30871606), the Major Science and Technology Project to Create New Crop Cultivars using Gene Transfer Technology (grant number 2014ZX08001-002), the Special Fund for AgroScientific Research in the Public Interest Program of China (grant number 20120314), and National Sci-tech Support Plan (2013BAD01B02-02-02).

\section{LITERATURE CITED}

Bryan, G. T., Wu, K. S., Farrall, L., Jia, Y., Hershey, H. P., McAdams, S. A., Faulk, K. N., Donaldson, G. K., Tarchini, R., and Valent, B. 2000. A single amino acid difference distinguishes resistant and susceptible alleles of the rice blast resistance gene Pi-ta. Plant Cell 12:2033-2046.
Chai, R., and Jin, M. 1995. Preliminary studies on the techniques for the in vitro inoculation of blast fungus. Acta Agr. Zhejiangensis 7:488.

Chen, J., Shi, Y., Liu, W., Chai, R., Fu, Y., Zhuang, J., and Wu, J. 2011. A Pid3 allele from rice cultivar Gumei 2 confers resistance to Magnaporthe oryzae. J. Genet. Genomics 38:209-216.

Chiu, W., Niwa, Y., Zeng, W., Hirano, T., Kobayashi, H., and Sheen, J. 1996. Engineered GFP as a vital reporter in plants. Curr. Biol. 6:325-330.

Collaborative Group for Identification of Yunnan Rice Germplasm Resources for Resistance to Diseases and Pests. 1992. Joint study on Yunan rice germplasm resources for their blast resistance. Crop Genet. Res. 4:28-30.

Fukuta, Y., Telebanco-Yanoria, M. J., Imbe, T., Tsunematsu, H., Kato, H., Ban, T., Ebron, L. A., Hayashi, N., Ando, I., and Khush, G. S. 2004 Monogenic lines as an international standard differential set for blast resistance in rice (Oryza sativa L.). Rice Genet. Newsl. 21:70-72.

Hao, Z., Wang, L., and Tao, R. 2009. Expression patterns of defence genes and antioxidant defence responses in a rice variety that is resistant to leaf blast but susceptible to neck blast. Physiol. Mol. Plant Pathol. 74:167-174

Hao, Z., Wang, L., Huang, F., and Tao, R. 2012. Expression of defense genes and antioxidant defense responses in rice resistance to neck blast at the preliminary heading stage and full heading stage. Plant Physiol. Biochem. 57:222-230.

Hayashi, N., Inoue, H., Kato, T., Funao, T., Shirota, M., Shimizu, T., Kanamori, H., Yamane, H., Hayano-Saito, Y., Matsumoto, T., Yano, M. and Takatsuji, H. 2010. Durable panicle blast-resistance gene $\mathrm{Pbl}$ encodes an atypical CC-NBS-LRR protein and was generated by acquiring a promoter through local genome duplication. Plant J. 64:498-510.

Hiei, Y., Ohta, S., Komari, T., and Kumashiro, T. 1994. Efficient transformation of rice (Oryza sativa L.) mediated by Agrobacterium and sequence analysis of the boundaries of the T-DNA. Plant J. 6 : 271-282.

Hittalmani, S., Parco, A., Mew, T. W., Ziegler, R. S., and Huang, N. 2000. Fine mapping and DNA marker-assisted pyramiding of the three major genes for blast resistance in rice. Theor. Appl. Genet. 100:1121-1128.

Hu, H. Y., Zhuang, J. Y., Chai, R. Y., Wu, J. L., Fan, Y. Y., and Zheng, K. L. 2006. Isolation and characterization of defense response genes involved in neck blast resistance of rice. Acta Genetica Sinica 33:251-261.

Huang, H., Huang, L., Feng, G., Wang, S., Wang, Y., Liu, J., Jiang, N., Yan, W., Xu, L., Sun, P., Li, Z., Pan, S., Liu, X., Xiao, Y., Liu, E., Dai, L., and Wang, G. L. 2011. Molecular mapping of the new blast resistance genes Pi47 and Pi48 in the durably resistant local rice cultivar Xiangzi 3150. Phytopathology 101:620-626.

Inoue, H., Higuchi, K., Takahashi, M., Nakanishi, H., Mori, S., and Nishizawa, N. K. 2003. Three rice nicotianamine synthase genes, OsNAS1, OsNAS2, and $O s N A S 3$ are expressed in cells involved in long-distance transport of iron and differentially regulated by iron. Plant J. 36:366-381.

International Rice Genome Sequencing Project. 2005. The map-based sequence of the rice genome. Nature 436:793-800.

International Rice Research Institute. 2002. Page 56 in: Standard Evaluation System for Rice. International Rice Research Institute, Manila, The Philippines.

Ishihara, T., Hayano-Saito, Y., Oide, S., Ebana, K., La, N. T., Hayahi, K., Ashizawa, T., Suzuki, F., and Koizumi, S. 2014. Quantitative trait locus analysis of resistance to panicle blast in the rice cultivar Miyazakimochi. Rice 7:2. www.thericejournal.com/content/7/1/2. Published online.

Jia, Y. 2003. Marker assisted selection for the control of rice blast disease. Pesticide Outlook 14:150-152.

Jones, J. D., and Dangl, J. L. 2006. The plant immune system. Nature 444: 323-329.

Kobayashi, N., Yanoria, M. J. T., and Fukuta, Y. 2007. Differential varieties bred at IRR and virulence analysis of blast isolates from the Philippines. JIRCAS Working Rep. 53:17-30.

Lei, C., Wang, J., Mao, S., Jiang, W., Zhu, L., and Ling, Z. 1996. Genetic analysis of blast resistance in indica variety Zhaiyeqing 8 (ZYQ8). Chin. J. Genet. 23:287-293.

Lei, C., Hao, K., Yang, Y., Ma, J., Wang, S., Wang, J., Cheng, Z., Zhao, S., Zhang, X., Guo, X., Wang, C., and Wan, J. 2013. Identification and fine mapping of two blast resistance genes in rice cultivar 93-11. Crop J. 1: 2-14.

Li, W., Lei, C., Cheng, Z., Jia, Y., Huang, D., Wang, J., Zhang, X., Su, N., Guo, X., Zhai, H., and Wan, J. 2008. Identification of SSR markers for a broad-spectrum blast resistance gene Pi2O(t) for marker-assisted breeding. Mol. Breed. 22:141-149.

Li, X., Lan, B., Chen, Q., Huang, L., Zhang, T., and Wang, R. 2010. Comparison of three identification methods of rice resistance to neck blast. Acta Phytophylacica Sin. 37:569-570.

Lin, F., Chen, S., Que, Z., Wang, L., Liu, X., and Pan, Q. 2007. The blast resistance gene Pi37 encodes a nucleotide binding site leucine-rich 
repeat protein and is a member of a resistance gene cluster on rice chromosome 1. Genetics 177:1871-1880.

Liu, J., Liu, X., Dai, L., and Wang, G. 2007. Recent progress in elucidating the structure, function and evolution of disease resistance genes in plants. J. Genet. Genomics 34:765-776.

Liu, J., Wang, X., Mitchell, T., Hu, Y., Liu, X., Dai, L., and Wang, G. L. 2010. Recent progress and understanding of the molecular mechanisms of the rice-Magnaporthe oryzae interaction. Mol. Plant Pathol. 11:419-427.

Liu, Y., Liu, B., Zhu, X., Yang, J., Bordeos, A., Wang, G., Leach, J. E., and Leung, H. 2013. Fine-mapping and molecular marker development for Pi56(t), a NBS-LRR gene conferring broad-spectrum resistance to Magnaporthe oryzae in rice. Theor. Appl. Genet. 126:985-998.

Livak, K. J., and Schmittgen, T. D. 2001. Analysis of relative gene expression data using real-time quantitative PCR and the $2-\Delta \Delta C T$ method. Methods. 25: 402-408.

Luo, S., Peng, J., Li, K., Wang, M., and Kuang, H. 2011. Contrasting evolutionary patterns of the $R p 1$ resistance gene family in different species of Poaceae. Mol. Biol. Evol. 28:313-325.

Mao, B., Cheng, Z., Lei, C., Xu, F., Gao, S., Ren, Y., Wang, J., Zhang, X., Wang, J., Wu, F., Guo, X., Liu, X., Wu, C., Wang, H., and Wan, J. 2012. Wax crystal-sparse leaf 2 , a rice homologue of $W A X 2 / G L 1$, is involved in synthesis of leaf cuticular wax. Planta 235:39-52.

Martin, G. B., Bogdanove, A. J., and Sessa, G. 2003. Understanding the functions of plant disease resistance proteins. Annu. Rev. Plant Biol. 54:23-61.

McCouch, S. R. 2008. Gene nomenclature system for rice. Rice 1:72-84.

McCouch, S. R., Teytelman, L., Xu, Y., Lobos, K. B., Clare, K., Walton, M., Fu, B., Maghirang, R., Li, Z., Xing, Y., Zhang, Q., Kono, I., Yano, M., Fjellstrom, R., DeClerck, G., Schneider, D., Cartinhour, S., Ware, D., and Stein, L. 2002. Development and mapping of 2240 new SSR markers for rice (oryza sativa L.). DNA Res. 9:199-207.

McDonnell, A. V., Jiang, T., Keating, A. E., and Berger, B. 2006. Paircoil2: Improved prediction of coiled coils from sequence. Bioinformatics 22: 356-358.

Michelmore, R. W., Paran, I., and Kesseli, R. V. 1991. Identification of markers linked to disease-resistance genes by bulked segregant analysis: A rapid method to detect markers in specific genomic regions by using segregating populations. Proc. Natl. Acad. Sci. USA 88:9828-9832.

Murray, M. G., and Thompson, W. F. 1980. Rapid isolation of high molecular weight plant DNA. Nucleic Acids Res. 8:4321-4325.

Puri, K. D., Shrestha, S. M., Chhetri, G. B. K., and Joshi, K. D. 2009. Leaf and neck blast resistance reaction in tropical rice lines under greenhouse condition. Euphytica 165:523-532.

Qi, D., DeYoung, B. J., and Innes, R. W. 2012. Structure-function analysis of the coiled-coil and leucine-rich repeat domains of the RPS5 disease resistance protein. Plant Physiol. 158:1819-1832.

Qu, S., Coaker, G., Francis, D., Zhou, B., and Wang, G. L. 2003. Development of a new transformation-competent artificial chromosome (TAC) vector and construction of tomato and rice TAC libraries. Mol. Breed. 12:297-308

Rao, Z. M., Wu, J. L., Zhuang, J. Y., Chai, R. Y., Fan, Y. Y., Leung, H., and Zheng, K. L. 2005. Genetic dissections of partial resistances to leaf and neck blast in rice (Oryza sativa L.). Acta Genetica Sin. 32:555-565.

Richter, T. E., and Ronald, P. C. 2000. The evolution of disease resistance genes. Plant Mol. Biol. 42:195-204.

Roumen, E. C. 1992. Partial resistance to neck blast influenced by stage of panicle development and rice genotype. Euphytica 64:173-182.

RoyChowdhury, M., Jia, Y., and Cartwright, R. D. 2012. Structure, function, and co-evolution of rice blast resistance genes. Acta Agron. Sin. 38:381-393.

Shi, K., Lei, C., Cheng, Z., Xu, X., Wang, J., and Wan, J. 2009. Distribution of two blast resistance genes Pita and Pib in major rice cultivars in China. J. Plant Genet. Resour. 10:21-26.

Sirithunya, P., Tragoonrung, S., Vanavichit, A., Pa-In, N., Vongsaprom, C., and Toojinda, T. 2002. Quantitative trait loci associated with leaf and neck blast resistance in recombinant inbred line population of rice (Oryza sativa). DNA Res. 9:79-88.

Sun, G., and Su, S. 1993. Studies on the application of a smearing inoculation technique in screening of neck blast in rice. Jiangxi Plant Prot. 16:21-23.

Sun, G., Su, S., and Shen, Z. 1992. A new inoculation technique for neck blast on in vitro rice panicle. Chinese J. Rice Sci. 6:39-42.

Takahashi, A., Hayashi, N., Miyao, A., and Hirochika, H. 2010. Unique features of the rice blast resistance Pish locus revealed by large scale retrotransposon-tagging. BMC Plant Biol. 10:175.

Tamura, K., Dudley, J., Nei, M., and Kumar, S. 2007. MEGA4: Molecular Evolutionary Genetics Analysis (MEGA) software version 4.0. Mol. Biol. Evol. 24:1596-1599.

Tan, X. H., Chen, X. W., Li, S. G., Liu, S. G., and Zhu, L. H. 2000. [Gene analysis of blast resistance in an indica variety Digu]. Acta Genetica Sinica 27:701-705.
Toki, S., Hara, N., Ono, K., Onodera, H., Tagiri, A., Oka, S., and Tanaka, H. 2006. Early infection of scutellum tissue with Agrobacterium allows high-speed transformation of rice. Plant J. 47:969-976.

Traut, T. W. 1994. The functions and consensus motifs of nine types of peptide segments that form different types of nucleotide-binding sites. Eur. J. Biochem. 222:9-19.

Tsunematsu, H., Yanoria, M. J. T., Ebron, L. A., Hayashi, N., Ando, I., Kato, H., Imbe. T., and Khush, G. S. 2000. Development of monogenic lines of rice for blast resistance. Breed. Sci. 50: 229-234.

van der Biezen, E. A., and Jones, J. D. 1998. The NB-ARC domain: A novel signalling motif shared by plant resistance gene products and regulators of cell death in animals. Curr. Biol. 8:R226-R227.

Wang, Z. X., Yano, M., Yamanouchi, U., Iwamoto, M., Monna, L., Hayasaka, H., Katayose, Y., and Sasaki, T. 1999. The Pib gene for rice blast resistance belongs to the nucleotide binding and leucine-rich repeat class of plant disease resistance genes. Plant J. 19:55-64.

Wicker, T., Sabot, F., Hua-Van, A., Bennetzen, J. L., Capy, P., Chalhoub, B., Flavell, A., Leroy, P., Morgante, M., Panaud, O., Paux, E., SanMiguel, P. and Schulman, A. H. 2007. A unified classification system for eukaryotic transposable elements. Nat. Rev. Genet. 8:973-982.

Wu, J., Zhuang, J., Cai, R., Fan, Y., Jin, M., Li, D., and Zheng, K. 2000. Molecular mapping of a gene conferring resistance to neck blast in rice. Acta Phytopathologica Sin. 30:112-115.

Wu, S., Zhu, X., Liu, B., Yang, Q., Zhang, S., and Leung, H. 2004. Genetic analysis and evaluation of durable resistance to blast in indica cultivar Sanhuangzhan 2. Scientia Agri. Sin. 37:528-534.

Wu, J. L., Fan, Y. Y., Li, D. B., Zheng, K. L., Leung, H., and Zhuang, J. Y. 2005. Genetic control of rice blast resistance in the durably resistant cultivar Gumei 2 against multiple isolates. Theor. Appl. Genet. 111:50-56.

Wu, J., Liu, X., Dai, L., and Wang, G. L. 2007. Advances on the identification and characterization of broad-spectrum blast resistance genes in rice. Sci. China Life Sci. 19:233-238.

Yang, Y., Wu, J., Chen, Z., Wang, L., Guo, H., Li, J., Liu, X., and Lu, Y. 2009. Mining rice new germplasm containing $S_{5}{ }^{n}$ gene by functional molecular marker and sequencing. Chin. Sci. Bull. 54:2212-2218.

Zeigler, R. S., Tohme, J., Nelson, R., Levy, M., and Correa Victoria, F. J. 1994. Lineage exclusion: A proposal for linking blast population analysis to resistance breeding. Pages 267-292.in: Rice blast disease, R. S. Zeigler, S. A. Leong, and P. S. Teng, eds. CAB international, Wallingford, U.K.

Zhai, C., Lin, F., Dong, Z., He, X., Yuan, B., Zeng, X., Wang, L., and Pan, Q. 2011. The isolation and characterization of Pik, a rice blast resistance gene which emerged after rice domestication. New Phytol. 189:321-334

Zhou, B., Qu, S., Liu, G., Dolan, M., Sakai, H., Lu, G., Bellizzi, M., and Wang, G. L. 2006. The eight amino-acid differences within three leucine-rich repeats between $P i 2$ and $P i z$ - $t$ resistance proteins determine the resistance specificity to Magnaporthe grisea. Mol. Plant Microbe Interact. 19:1216-1228.

Zhou, B., Dolan, M., Sakai, H., and Wang, G. L. 2007. The genomic dynamics and evolutionary mechanism of the Pi2/9 locus in rice. Mol Plant-Microbe Interact. 20:63-71.

Zhu, X., Chen, S., Yang, J., Zhou, S., Zeng, L., Han, J., Su, J., Wang, L., and Pan, Q. 2012. The identification of Pi5O(t), a new member of the rice blas resistance Pi2/Pi9 multigene family. Theor. Appl. Genet. 124:1295-1304

Zhuang, J., Ma, W., Wu, J., Chai, R., Lu, J., Fan, Y., Jin, M., Leung, H., and Zheng, K. 2002. Mapping of leaf and neck blast resistance genes with resistance gene analog, RAPD and RFLP in rice. Euphytica 128:363-370.

Zipfel, C. 2008. Pattern-recognition receptors in plant innate immunity. Curr. Opin. Immunol. 20:10-16.

\section{AUTHOR-RECOMMENDED INTERNET RESOURCE}

Cambia website: www.cambia.org

dCAPS Finder 2.0 software: helix.wustl.edu/dcaps/dcaps.html

EMBL SMART tool: smart.embl-heidelberg.de

ExPASy Bioinformatics Resource portal: us.expasy.org/cgi-bin

Gramene database: www.gramene.org

International Rice Genome Sequencing Project database: rgp.dna.affrc.go.jp

Massachusetts Institute of Technology Scansite database: scansite.mit.edu

Michigan State University Rice Genome Annotation Project database: rice.plantbiology.msu.edu

National Center for Biotechnology Information (NCBI) database: www.ncbi.nlm.nih.gov

NCBI BLAST database: www.ncbi.nlm.nih.gov/BLAST

Paircoil2 program: groups.csail.mit.edu/cb/paircoil2

SoftBerry website: linux1.softberry.com 